\title{
Reversible Cerebral Vasoconstriction Syndrome Associated with Infundibular Dilation
}

\author{
Héctor Montenegro-Rosales ${ }^{1}$, Blanca González-Alonso ${ }^{1}$, Omar Cárdenas-Sáenz², Alonso Gutierrez-Romero³ \\ ${ }^{1}$ Medica Sur Hospital, Magnetic Resonance Imaging Department, Mexico City, Mexico \\ ${ }^{2}$ ISSSTE Zaragoza, Neurology Department, Mexico City, Mexico \\ ${ }^{3}$ Medica Sur Hospital, Neurology Department, Mexico City, Mexico
}

Received: 26/06/2020

Accepted: $30 / 06 / 2020$

Published: $31 / 08 / 2020$

\begin{abstract}
How to cite this article: Montenegro-Rosales H, González-Alonso B, Cárdenas-Sáenz O, Gutierrez-Romero A. Reversible cerebral vasconstriction syndrome associated with infudibular dilation. EJCRIM 2020;7: doi:10.12890/2020_001839.
\end{abstract}

Conflicts of Interests: The Authors declare that there are no competing interests.

This article is licensed under a Commons Attribution Non-Commercial 4.0 License

\section{ABSTRACT}

Background: Reversible cerebral vasoconstriction syndrome (RCVS) is defined as a clinical and radiological syndrome that comprises a group of disorders characterized by sudden-onset severe headache and segmental vasoconstriction of the cerebral arteries with resolution within 3 months.

Case presentation: A 51-year-old female patient with a 2-week history of sudden-onset severe headache, visual disturbances and cerebellum; no relevant imaging findings, except for an infundibular dilation at the origin of the posterior communicating artery, and so, angiography was performed. When symptoms persisted, a new imaging study was carried out with findings of RCVS as the cause of the symptoms from the beginning.

Conclusions: Findings of RCVS can be obtained in various vasculopathies of the nervous system and vasculitis, being misdiagnosed, and so, clinical suspicion is essential; if vasoconstriction is not demonstrated on the initial image and other diagnoses have been excluded, the patient should be managed as having possible or probable RCVS.

\section{LEARNING POINTS}

- RCVS is a clinical and radiological syndrome that comprises a group of disorders characterized by sudden-onset severe headache and segmental vasoconstriction of the cerebral arteries.

- It can occur spontaneously or can be triggered by a specific "trigger" in 25 to $60 \%$ of cases.

- It commonly affects women between 20 and 50 years of age, but children and adolescents may also be affected.

\section{KEYWORDS}

Reversible cerebral vasoconstriction syndrome, sudden-onset severe headache

\section{CASE DESCRIPTION}

A 51-year-old woman with a history of a prolactin-producing pituitary microadenoma and taking oxymetazoline in a nasal spray experienced a burst headache while evacuating, with an intensity of 10 out of 10 in less than 1 minute. It was holocranial, pulsatile and accompanied by nausea without vomiting, radiation, exacerbation or mitigation of symptoms. The same headache was experienced 3 more times, triggered by the Valsalva manoeuvre. Due to the symptoms, the patient went to the local hospital where computed tomographic angiography (CTA) and magnetic resonance angiography (MRA) was performed with no pathological findings (Fig. 1); consequently, she was sent home with oral non-specified analgesic treatment. 


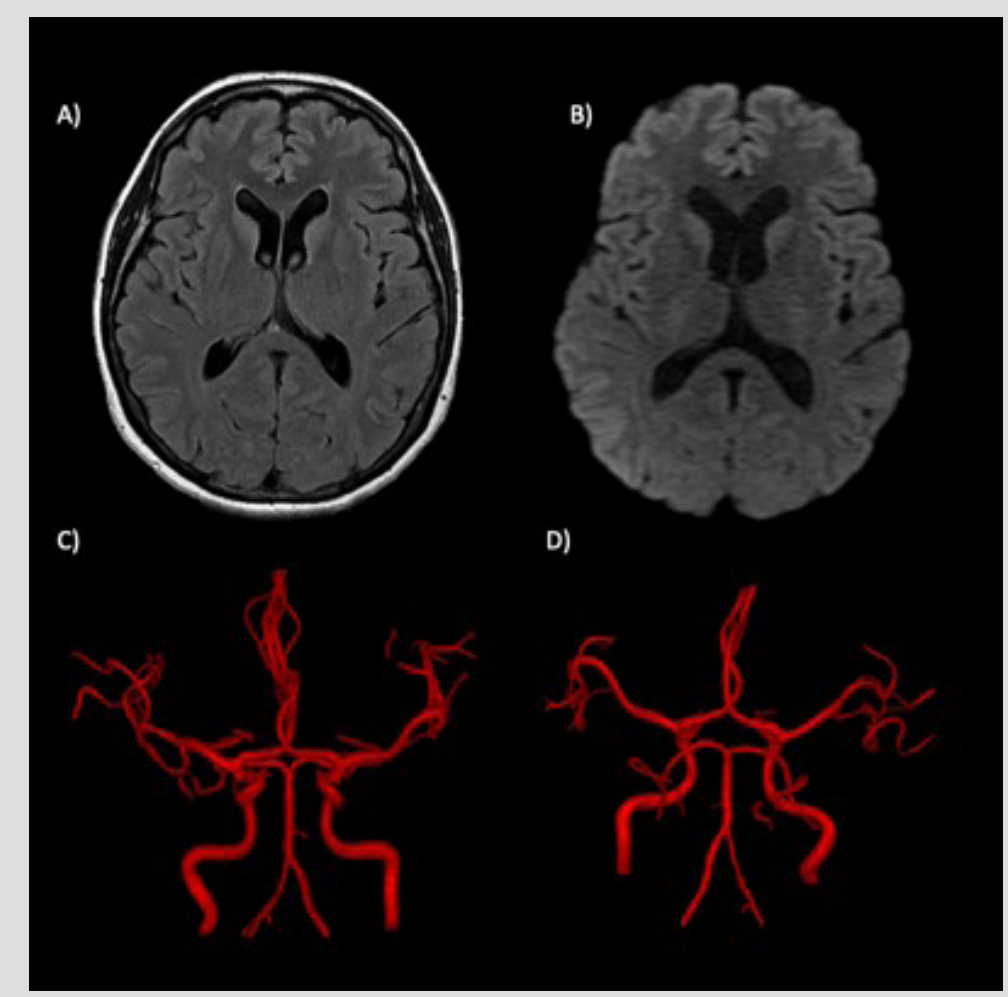

Figure 1. Initial MRA findings. (A) FLAIR and (B) diffusion imaging. No alterations of the brain parenchyma were identified. (C) Anterior and (D) posterior views of 3D TOF volumetric reconstructions. The calibre of the intracranial arteries was normal

For 2 weeks the patient experienced episodes of intermittent headache with no improvement after analgesic treatment.

A new MRA assessment was carried out to rule out pituitary stroke or subarachnoid haemorrhage, finding an infundibular dilation at the origin of the posterior communicating artery in the left internal carotid artery, while the calibre of the rest of the vessels of the anterior and posterior circulation was normal in all evaluated segments. Two days later and given these findings, the patient underwent diagnostic cerebral angiography and stent placement to cover the infundibular dilation at the origin of the posterior communicating artery in the left internal carotid artery; during the procedure, nimodipine $(3,000 \mathrm{mg})$ and heparin (1,000 units) were administered, with no changes in the calibre of the vessels of the anterior and posterior circulation (Fig. 2).

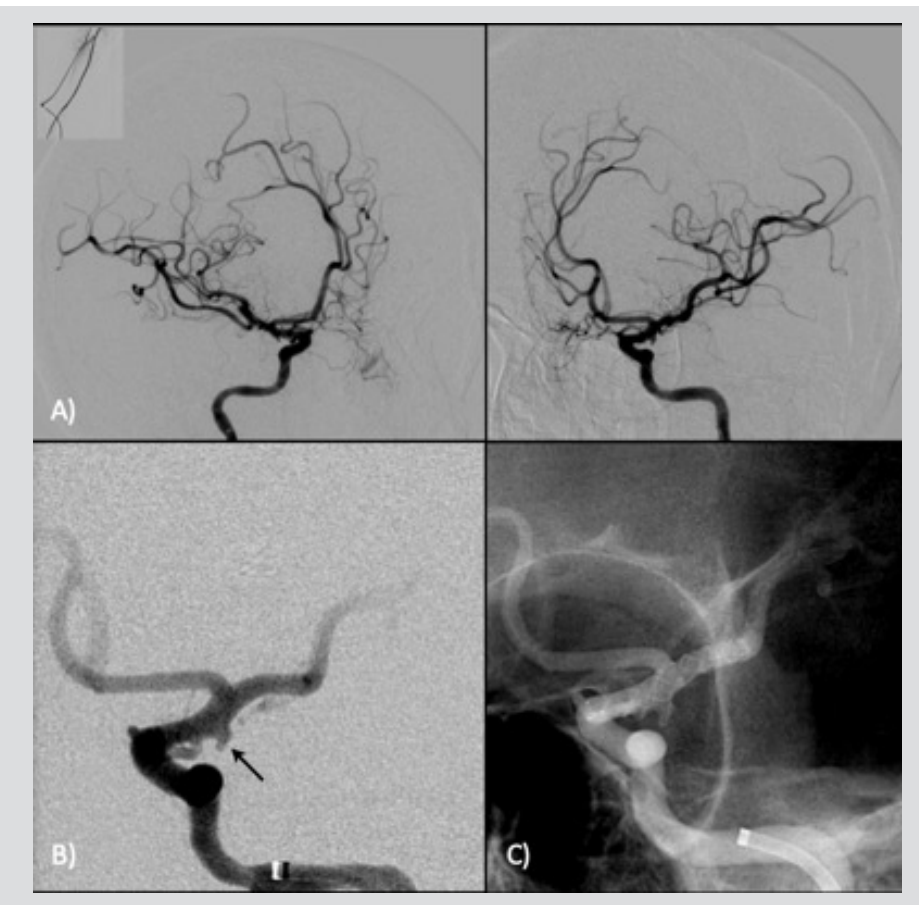

Figure 2. Angiography; radial approach. (A) Oblique images of the left and right internal carotid arteries; the calibre of the intracranial arteries was normal. (B) Infundibular dilation at the origin of the left posterior communicating artery (arrow). (C) Postplacement of the LVIS stent without complications 
One day later, the patient presented mild headache, intensity 4 out of 10 and holocranial, and fatigue, without any neurological targeting data; hours later, she presented drowsiness, disorientation in time, space, person and circumstance, dysarthria and semantic paraphasia, as well as sensory dysphasia, and so, a new simple CT scan was performed with no pathological findings. As the patient persisted with disorientation and paraphasia, MRI was performed, finding cortico-subcortical parietal and occipital hyperintensities predominantly on the right; in addition, MRA showed changes in the calibre of arterial vascular structures of both the anterior and posterior circulation and asymmetrically. Dexamethasone $8 \mathrm{mg}$ was initiated every 8 hours and a complete rheumatologic profile and electroencephalogram were requested, showing abnormal wakefulness due to alterations indicative of mild diffuse subcortical and cortical dysfunction with greater left temporo-occipital expression and increased left frontal-temporal cortico-subcortical excitability. On physical examination the patient was drowsy, inattentive and disoriented, with dysarthric speech, semantic paraphasia, emotional lability and easy crying; cranial nerve assessment findings included visual acuity 20/100, left homonymous hemianopia and Weber lateralized to the right, Rinne negative on the left side (hearing loss left). Decreased tone in the left side of the body, dysmetria and dysdiadochokinesia on the same side were also observed.

The patient presented significant improvement in symptoms, recovering visual acuity with restoration of vision within the visual field, without drowsiness, dysarthria or semantic paraphasia; only with headache, dysmetria and left dysdiadochokinesia.

Three days later, new MRI showed an increase in the size of the described lesions and new lesions in sites of the anterior circulation with generalized vasospasm observed in 3D TOF MRA images, predominantly in the posterior circulation (Fig. 3). As the signs on control MRI were getting worse, it was concluded that the patient had reversible cerebral vasoconstriction syndrome (RCVS), which was the cause of all the headache outbreaks that she presented from the beginning.

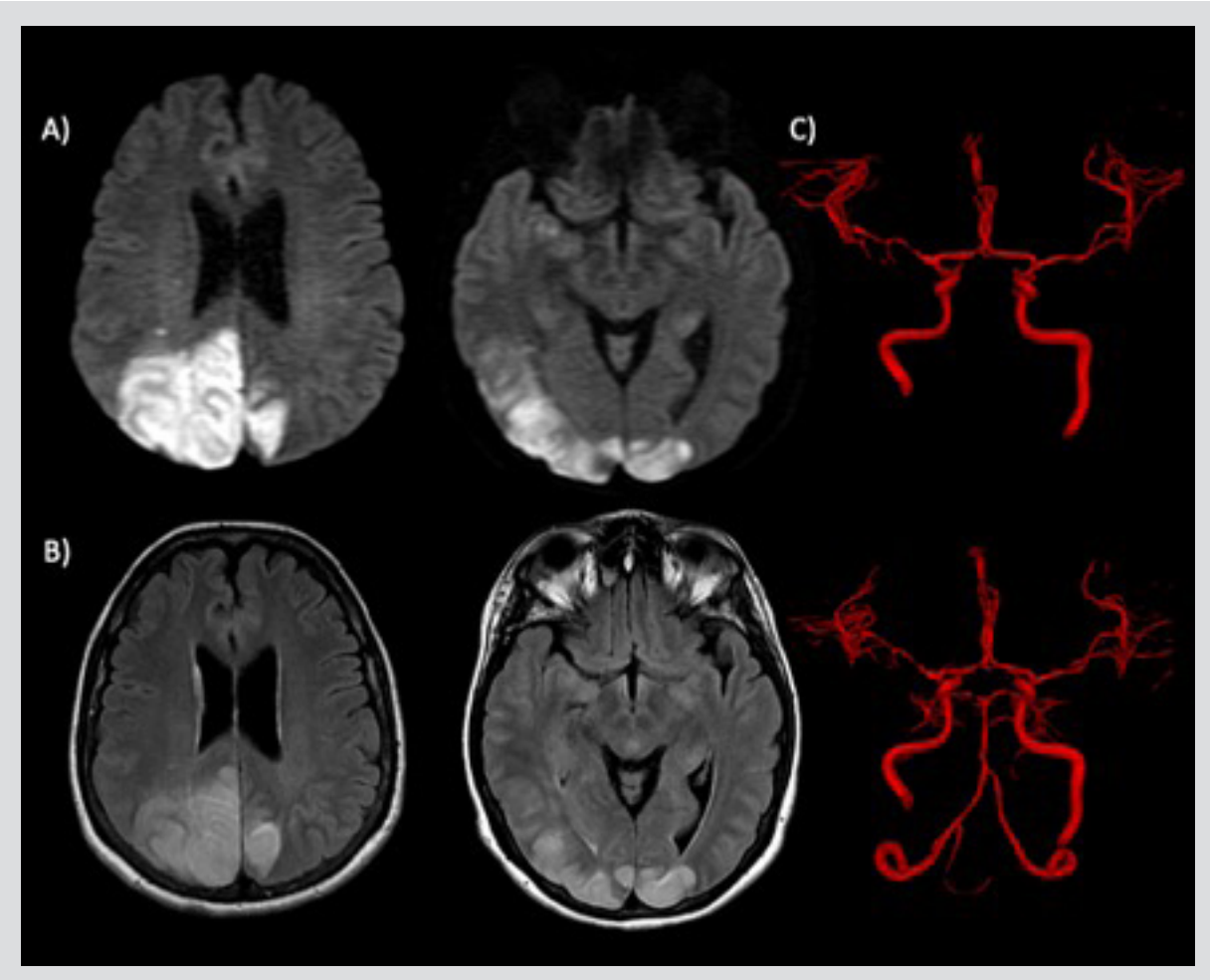

Figure 3. Control MRA. (A) Diffusion and (B) FLAIR imaging. Oedema affecting grey-white matter, bilateral and asymmetrical, with predominance in the right hemisphere. (C) Anterior and posterior view of 3D TOF volumetric reconstructions. There was spasm of all intracranial arterial vascular territories

Once the aetiological diagnosis was integrated, the doses of dexamethasone were decreased and verapamil 80 mg every 8 hours was administered. Forty-eight hours after initiation of verapamil, a significant improvement in the generalized vasospasm was observed, which was not visualized with the depot steroid (Fig. 4); the residual headache disappeared, and improvement in visual acuity and in left cerebellar hemispheric syndrome was seen.

\section{DISCUSSION}

RCVS is defined as a clinical and radiological syndrome characterized by intense headache of sudden onset and segmental vasoconstriction of the cerebral arteries ${ }^{[1,2]}$ that resolves in 3 months and encompasses a wide variety of disorders previously described by other names ${ }^{[1,3-7]}$. 


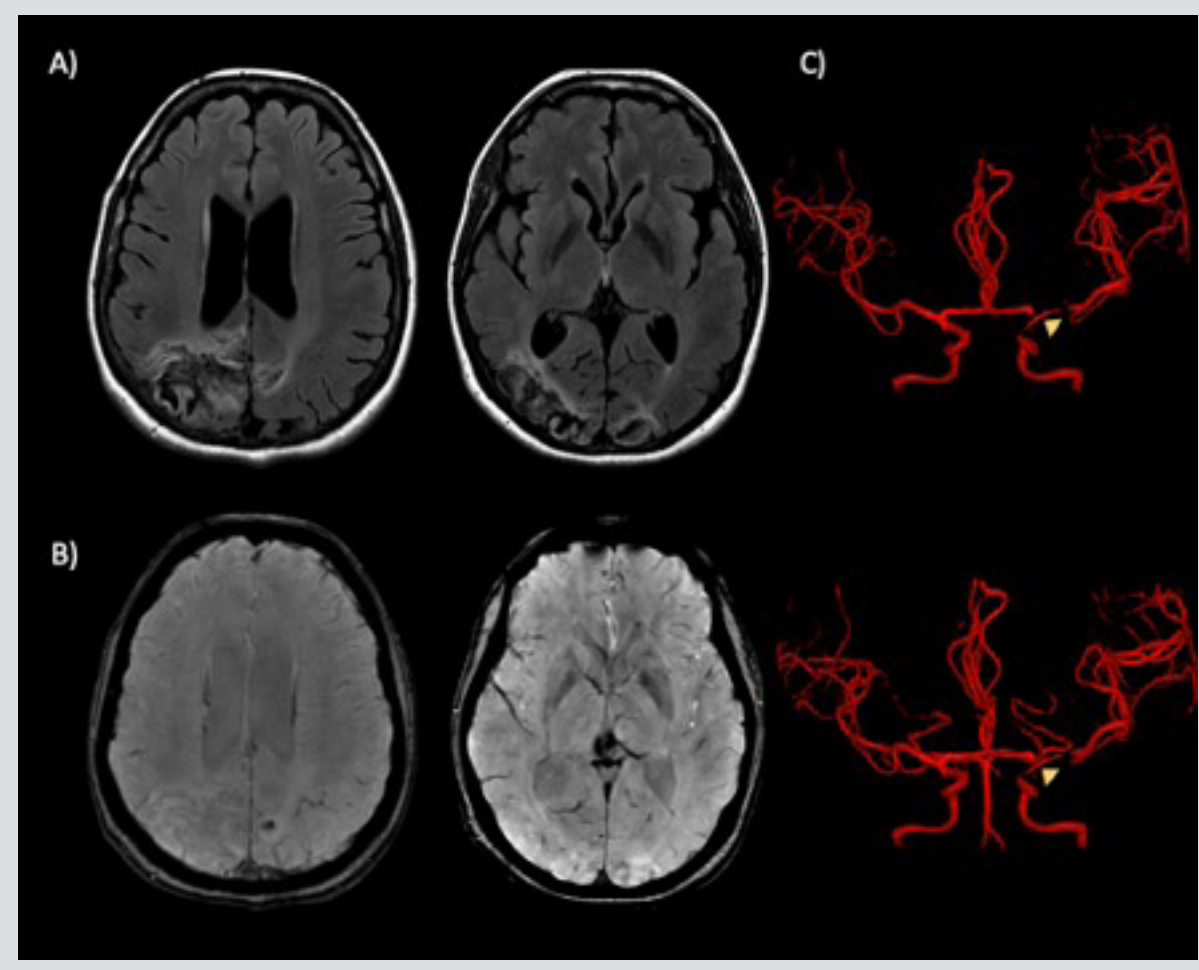

Figure 4. Post-treatment MRA. (A) FLAIR and (B) SWAN imaging. Bilateral areas of encephalomalacia with haemosiderin deposits can be seen; there were no restriction zones on diffusion imaging (image not shown). (C) 3D TOF volumetric reconstructions. Adequate calibre of intracranial arterial structures was observed; only a thinned path (arrowheads) of the posterior communicating segment of the carotid was seen due to the presence of the stent

Diagnostic imaging findings may be evident on angiography, CT, MRI or transcranial Doppler (TCD) and include multifocal segmental constrictions alternating with focal dilations or normal-calibre vessels in multiple vascular distributions; in accordance with Marder et al. ${ }^{[5]}$, in some cases the initial vascular imaging findings may be normal, with abnormalities developing over days to weeks, as in the case presented here ${ }^{[9]}$.

The differential diagnosis includes PRES, subarachnoid haemorrhage due to rupture of the aneurysm, primary angiitis of the central nervous system, cortical venous thrombosis, migraine headache and an ischaemic cerebral vascular event.

RCVS is considered to be a painful but benign syndrome; however, potential complications may develop, such as infarctions and intracranial haemorrhages as reported by Ducros et al. ${ }^{[8]}$, including cortical subarachnoid haemorrhages, intracerebral haemorrhages and subarachnoid haemorrhage, as reported by Ansari et al. ${ }^{[3]}$. This is the reason why early recognition of RCVS allows appropriate clinical management aimed at reducing the frequency and severity of vascular complications, by identification and elimination of the causative vasoactive agent or stimulus and short-term treatment with a calcium channel antagonist, as in this case ${ }^{[10]}$.

\section{REFERENCES}

1. Miller TR, Shivashankar R, Mossa-Basha M, Gandhi D. Reversible cerebral vasoconstriction syndrome, part 1: epidemiology, pathogenesis, and clinical course. Am J Neuroradio 2015;36(8):1392-1399.

2. Bartynski WS. Posterior reversible encephalopathy syndrome, part 2: controversies surrounding pathophysiology of vasogenic edema. Am J Neuroradiol 2008;29(6):10431049.

3. Ansari SA, Rath TJ, Gandhi D. Reversible cerebral vasoconstriction syndromes presenting with subarachnoid hemorrhage: a case series. J Neurointerv Surg 2011;3(3):272-278.

4. Chen SP, Fuh JL, Wang SJ, Chang FC, Lirng JF, Fang YC, et al. Magnetic resonance angiography in reversible cerebral vasoconstriction syndromes. Ann Neurol 2010;67(5):648656.

5. Marder CP, Donohue MM, Weinstein JR, Fink KR. Multimodal imaging of reversible cerebral vasoconstriction syndrome: a series of 6 cases. Am J Neuroradiol 2012;33(7):14031410.

6. Calabrese LH, Dodick DW, Schwedt TJ, Singhal AB. Narrative review: reversible cerebral vasoconstriction syndromes. Ann Intern Med 2007;146(1):34-44.

7. Burton TM, Bushnell CD. Reversible cerebral vasoconstriction syndrome: a diagnostic imaging review. Stroke 2019;50(8):2253-2258.

8. Ducros A, Fiedler U, Porcher R, Boukobza M, Stapf C, Bousser MG. Hemorrhagic manifestations of reversible cerebral vasoconstriction syndrome: frequency, features, and risk factors. Stroke 2010;41(11):2505-2511.

9. Bartynski WS. Posterior reversible encephalopathy syndrome, part 1: fundamental imaging and clinical features. Am J Neuroradiol 2008;29(6):1036-1042

10. Ducros A. Reversible cerebral vasoconstriction syndrome. Lancet Neurol 2012;11(10):906-917. 